\title{
Single crystal to single crystal [2+2] photoreactions in chloride and sulphate salts of 4-amino-cinnamic acid via solid-solution formation: a structural and kinetic study.
}

\author{
Simone d'Agostino, ${ }^{\mathrm{a}, *}$ Floriana Spinelli, ${ }^{\mathrm{a}}$ Elisa Boanini, ${ }^{\mathrm{a}}$ Dario Braga, ${ }^{\mathrm{a}}$ and Fabrizia Grepioni ${ }^{\mathrm{a}, *}$ \\ a Dipartimento di Chimica “G. Ciamician”, Università di Bologna, Via F. Selmi 2, 40126 Bologna, Italy.
}

\section{Table of Contents}

Synthesis 2

Crystal structure determination and refinement details 3

PXRD for $[\mathbf{1 H}] \mathrm{Cl},[\mathbf{1}]_{2}\left[\mathrm{SO}_{4}\right] \cdot \mathrm{H}_{2} \mathrm{O}(\mathrm{I}),[\mathbf{1}]_{\mathrm{BF}_{4}}(\mathrm{I}),[\mathbf{1} \mathrm{H}] \mathrm{BF}_{4}(\mathrm{II})$ and $[\mathbf{1 H}] \mathrm{PF}_{6} \quad 7$

Structure determination and refinement of $\left[1 \mathrm{H}^{-N} \mathrm{O}_{3}\right.$ from powder diffraction data 9

$\begin{array}{ll}\text { Hydrogen bonds in }[\mathbf{1 H}] \mathrm{Cl} & 10\end{array}$

$\begin{array}{ll}\text { Hydrogen bonds in }[\mathbf{1 H}] \mathrm{NO}_{3} & 10\end{array}$

Hydrogen bonds in $[\mathbf{1 H}]_{2}\left[\mathrm{SO}_{4}\right] \cdot \mathrm{H}_{2} \mathrm{O}(\mathrm{I}) \quad 11$

Hydrogen bonds in $[\mathbf{1 H}]_{2}\left[\mathrm{SO}_{4}\right] \cdot \mathrm{H}_{2} \mathrm{O}$ (II) $\quad 11$

Hydrogen bonds in $[\mathbf{1 H}] \mathrm{BF}_{4}(\mathrm{I}) \quad 12$

Hydrogen bonds in $[\mathbf{1 H}] \mathrm{BF}_{4}(\mathrm{II}) \quad 12$

$\begin{array}{ll}\text { Hydrogen bonds in }[\mathbf{1 H}] \mathrm{PF}_{6} & 13\end{array}$

Geometrical parameters used for the evaluation of photoreactivity 13

Solid State Photoreaction $\quad 13$

Hydrogen bond variations over the course of photoreactions of $\left[1 \mathrm{H}_{2}\left[\mathrm{SO}_{4}\right] \cdot \mathrm{H}_{2} \mathrm{O}\right.$ (II) 15

Picture of single crystals of $[1 \mathrm{H}] \mathrm{Cl}$ and $[1 \mathrm{H}]_{2}\left[\mathrm{SO}_{4}\right] \cdot \mathrm{H}_{2} \mathrm{O}$ (II) before and after the photoreaction and crystal morphology for $[\mathbf{1 H}] \mathrm{Cl} \quad 16$

$\begin{array}{ll}\text { Scanning Electron Microscopy } & 17\end{array}$

Plots of the variation of cell parameters during photoreaction for $[\mathbf{1 H}] \mathrm{Cl}$ and $\left[1 \mathrm{H}_{2}\left[\mathrm{SO}_{4}\right] \cdot \mathrm{H}_{2} \mathrm{O}\right.$ (II) $\quad 17$

ATR-FTIR spectra of $[\mathbf{1 H}] \mathrm{Cl},[\mathbf{1 H}] \mathrm{NO}_{3}$ and $\left[\mathbf{1} \mathrm{H}^{-B_{4}}\right.$ (II) before and after UV irradiation $\quad 18$

NMR spectroscopy of $[\mathbf{1 H}] \mathrm{Cl}$ before and after UV irradiation $\quad 18$

References $\quad 19$ 
Synthesis. All reactants and reagents were purchased from Sigma-Aldrich and used without further purification. Reagent grade solvents and bi-distilled water were used. Synthesis is based on the reaction between 4-aminocinnamic acid (1) and $\mathrm{HCl}$ to obtain the chloride salt $[\mathbf{1 H}] \mathrm{Cl}$ followed by the anion exchange reaction of this with the appropriate silver salt used as halide abstraction reagent.

[1H]Cl: $200 \mathrm{mg}(1.23 \mathrm{mmol})$ of 4-aminocinnamic acid (1) were dissolved in aqueous $\mathrm{HCl}(\mathrm{PH}$ ca. 2), colorless crystals of $[\mathbf{1 H}] \mathrm{Cl}$ suitable for X-ray crystal diffraction were obtained from evaporation of the filtered aqueous solution over a period of 1 week.

$[1 \mathrm{H}] \mathrm{NO}_{3}: 120 \mathrm{mg}(0.72 \mathrm{mmol})$ of $\mathrm{AgNO}_{3}$ were dissolved in ca. $10 \mathrm{~mL}$ of EtOH, to the resulting solution were added $140 \mathrm{mg}(0.72 \mathrm{mmol})$ of $[1 \mathrm{H}] \mathrm{Cl}$ and kept in stirring in the dark for $5 \mathrm{~h}$. The precipitated $\mathrm{AgCl}$ was filtered off. A white powder of $[1 \mathbf{H}] \mathrm{NO}_{3}$ was obtained from slow evaporation of the solution over a period of 3 days.

$[1 \mathrm{H}]_{2} \mathrm{SO}_{4} \cdot \mathrm{H}_{2} \mathrm{O}: 200 \mathrm{mg}(0.64 \mathrm{mmol})$ of $\mathrm{Ag}_{2} \mathrm{SO}_{4}$ were dissolved in ca. $10 \mathrm{~mL}$ of water, to the resulting solution were added $130 \mathrm{mg}(0.64 \mathrm{mmol})$ of $[1 \mathrm{H}] \mathrm{Cl}$ and stirred in the dark for $5 \mathrm{~h}$. The precipitated $\mathrm{AgCl}$ was filtered off. Slow evaporation of the solution over a period of 1 week afforded crystals suitable for X-ray diffraction of both $\left[1 \mathrm{H}_{2} \mathrm{SO}_{4} \cdot \mathrm{H}_{2} \mathrm{O}(\mathrm{I})\right.$, and $[\mathbf{1 H}]_{2} \mathrm{SO}_{4} \cdot \mathrm{H}_{2} \mathrm{O}$ (II). Pure polycrystalline phase of polymorph (I) was obtained by seeding solutions of water, while it was not possible to obtain a pure phase for $[1 \mathrm{H}]_{2} \mathrm{SO}_{4} \cdot \mathrm{H}_{2} \mathrm{O}$ (II), which was invariably contaminated by Form I.

$[1 \mathrm{H}] \mathrm{BF}_{4}: 100 \mathrm{mg}(0.51 \mathrm{mmol})$ of $\mathrm{AgBF}_{4}$ were dissolved in ca.10 mL of water, to the resulting solution were added $100 \mathrm{mg}(0.5 \mathrm{mmol})$ of $[\mathbf{1 H}] \mathrm{Cl}$ and kept in stirring in the dark for $5 \mathrm{~h}$. The precipitated $\mathrm{AgCl}$ was filtered off. Slow evaporation of the solution over a period of 3 days afforded crystals suitable for X-ray diffraction of both $[\mathbf{1 H}] \mathrm{BF}_{4}(\mathrm{I})$, and $[\mathbf{1 H}] \mathrm{BF}_{4}$ (II). Pure polycrystalline phases of both polymorphs were obtained by seeding solutions of EtOH or water for $[\mathbf{1 H}] \mathrm{BF}_{4}(\mathrm{I})$ and $[\mathbf{1 H}] \mathrm{BF}_{4}(\mathrm{II})$, respectively.

[1H]PF $6: 100 \mathrm{mg}(0.4 \mathrm{mmol})$ of $\mathrm{AgPF}_{6}$ were dissolved in $c a .8 \mathrm{~mL}$ of water, to the resulting solution were added $80 \mathrm{mg}(0.4 \mathrm{mmol})$ of $[1 \mathrm{H}] \mathrm{Cl}$ and kept in stirring in the dark for $5 \mathrm{~h}$. The precipitated $\mathrm{AgCl}$ was filterd off. Yellow crystals of $[\mathbf{1} \mathrm{H}] \mathrm{PF}_{6}$ suitable for X-ray diffraction were obtained from slow evaporation of the solution over a period of 1 week. 
Crystal structure determination. Single-crystal data for all salts(except $\left[1 \mathrm{H}^{-} \mathrm{NO}_{3}\right.$; see below)were collected at RT on an Oxford X'Calibur S CCD diffractometer equipped with a graphite monochromator $\left(\mathrm{Mo}-\mathrm{K}_{\alpha}\right.$ radiation, $\lambda=0.71073 \AA$ ). Data collection and refinement details are listed in Tables1a, 1b, and 1c. All non-hydrogen atoms were refined anisotropically. $\mathrm{H}_{\mathrm{CH}}$ atoms for all compounds were added in calculated positions and refined riding on their respective carbon atoms; $\mathrm{H}_{\mathrm{OH}}$ and $\mathrm{H}_{\mathrm{NH}}$ atoms were either directly located or, when not possible, added in calculated positions. SHELX $97^{1}$ was used for structure solution and refinement on $\mathrm{F}^{2}$. The program Mercury ${ }^{2}$ was used to calculate intermolecular interactions. CYLview ${ }^{3}$ and Mercury ${ }^{2}$ were used for molecular graphics. Crystaldata can be obtained free of charge via www.ccdc.cam.ac.uk/conts/retrieving.htmL (or from the Cambridge Crystallographic Data Centre, 12 Union Road, Cambridge CB21EZ, UK; fax: (+44)1223-336-033; or e-mail: deposit@ccdc.cam.ac.uk). CCDC numbers 1436608-1436629. Crystal structures of partially reacted crystals were treated according to previously reported literature procedures, ${ }^{4,5}$ that is as follows: (i) upon irradiation, new electron density peaks, consistent with the emergence of the cyclobutane ring, appeared in the Delta Fourier Maps. The Catoms of the disappearing double bond and appearing cylclobutane were treated as disordered over two positions and refined isotropically, refinement of the site occupancy factors (SOFs) by adding a second free variable in the FVAR command line allowed the extraction of the dimer and reactant contents, (ii) the aminophenyl and carboxyllic side groups were modeled by convolving them into an average of two conformations.

From literature is known that [2+2] reactions do not revert back in ordinary conditions, ${ }^{6}$ on the other hand it has been observed in some metallorganic salts (MOSs) containing the photoreactive 4,4-stilbenedicarboxylic acid as building block that after exposure to UV $(\lambda<300 \mathrm{~nm})$ or after thermal treatment $\left(300^{\circ} \mathrm{C}\right)$, the photoreaction went back. ${ }^{7}$ In our case, we have determined the unit cell parameters, which are indicative of dimer content, twice and at time intervals of $8 \mathrm{~h}$ prior to full data collection. We have not noticed any unit cell variation between the two determined unit cells, thus concluding that the reaction had not gone back. 
Table 1a. Crystal data and details of measurements for crystalline $[1 \mathrm{H}] \mathrm{Cl}$ and for each stage of itsphotodimerization.

\begin{tabular}{|c|c|c|c|c|c|c|c|c|c|c|}
\hline & {$[1 \mathrm{H}] \mathrm{Cl}$} & {$[1 \mathrm{H}] \mathrm{Cl} \_86 \%$} & {$[1 \mathrm{H}] \mathrm{Cl} \_80 \%$} & {$[\mathbf{1 H}] \mathrm{Cl} \_74 \%$} & {$[1 \mathrm{H}] \mathrm{Cl} \_68 \%$} & {$[1 \mathrm{H}] \mathrm{Cl} \_51 \%$} & {$[1 \mathrm{H}] \mathrm{Cl} \_41 \%$} & {$[1 \mathrm{H}] \mathrm{Cl} \_37 \%$} & {$[1 \mathrm{H}] \mathrm{Cl} \_25 \%$} & {$[\mathbf{1 H}] \mathrm{Cl} \_0 \%$} \\
\hline Formula & $\mathrm{C}_{9} \mathrm{H}_{10} \mathrm{ClNO}_{2}$ & $\mathrm{C}_{9} \mathrm{H}_{10} \mathrm{ClNO}_{2}$ & $\mathrm{C}_{9} \mathrm{H}_{10} \mathrm{ClNO}_{2}$ & $\mathrm{C}_{9} \mathrm{H}_{10} \mathrm{ClNO}_{2}$ & $\mathrm{C}_{9} \mathrm{H}_{10} \mathrm{ClNO}_{2}$ & $\mathrm{C}_{9} \mathrm{H}_{10} \mathrm{ClNO}_{2}$ & $\mathrm{C}_{9} \mathrm{H}_{10} \mathrm{ClNO}_{2}$ & $\mathrm{C}_{9} \mathrm{H}_{10} \mathrm{ClNO}_{2}$ & $\mathrm{C}_{9} \mathrm{H}_{10} \mathrm{ClNO}_{2}$ & $\mathrm{C}_{9} \mathrm{H}_{10} \mathrm{ClNO}_{2}$ \\
\hline fw & 199.63 & 199.63 & 199.63 & 199.63 & 199.63 & 199.63 & 199.63 & 199.63 & 199.63 & 199.63 \\
\hline Cryst. System & Monoclinic & Monoclinic & Monoclinic & Monoclinic & Monoclinic & Monoclinic & Monoclinic & Monoclinic & Monoclinic & Monoclinic \\
\hline Space group & $\mathrm{P} 2{ }_{1} / \mathrm{c}$ & $\mathrm{P} 2{ }_{1} / \mathrm{c}$ & $\mathrm{P} 2{ }_{1} / \mathrm{c}$ & $\mathrm{P} 2{ }_{1} / \mathrm{c}$ & $\mathrm{P} 2{ }_{1} / \mathrm{c}$ & $\mathrm{P} 2{ }_{1} / \mathrm{c}$ & $\mathrm{P} 2{ }_{1} / \mathrm{c}$ & $\mathrm{P} 2{ }_{1} / \mathrm{c}$ & $\mathrm{P} 2{ }_{1} / \mathrm{c}$ & $\mathrm{P} 2{ }_{1} / \mathrm{c}$ \\
\hline $\mathrm{Z}$ & 4 & 4 & 4 & 4 & 4 & 4 & 4 & 4 & 4 & 4 \\
\hline $\mathrm{a}(\AA)$ & $5.5837(7)$ & $5.6063(2)$ & $5.6180(3)$ & $5.6294(5)$ & $5.6409(5)$ & $5.6523(7)$ & $5.6523(7)$ & $5.6907(5)$ & $5.7167(6)$ & 5.7431(17) \\
\hline $\mathrm{b}(\AA)$ & $8.5339(10)$ & $8.5811(7)$ & $8.5855(7)$ & $8.5824(8)$ & $8.5631(8)$ & $8.5616(14)$ & $8.5616(14)$ & $8.4732(18)$ & $8.417(2)$ & $8.221(3)$ \\
\hline $\mathrm{c}(\AA)$ & $19.865(2)$ & $19.4662(17)$ & $19.373(2)$ & $19.252(3)$ & 19.131(3) & $19.105(5)$ & $19.105(5)$ & $18.860(3)$ & 18.889(3) & $19.012(6)$ \\
\hline$\alpha(\operatorname{deg})$ & 90 & 90 & 90 & 90 & 90 & 90 & 90 & 90 & 90 & 90 \\
\hline$\beta(\operatorname{deg})$ & $92.074(11)$ & $91.595(7)$ & $91.521(7)$ & $91.427(9)$ & $91.475(10)$ & 91.471(17) & 91.471(17) & 91.741(9) & 91.904(10) & $92.47(3)$ \\
\hline$\gamma(\operatorname{deg})$ & 90 & 90 & 90 & 90 & 90 & 90 & 90 & 90 & 90 & 90 \\
\hline $\mathrm{V}\left(\AA^{3}\right)$ & $946.0(2)$ & 936.12(12) & $934.08(15)$ & 929.83(19) & 923.78(19) & $924.3(3)$ & 913.1(2) & $909.0(2)$ & 908.4(3) & $896.8(5)$ \\
\hline$D_{\text {calc }}\left(\mathrm{g} / \mathrm{cm}^{3}\right)$ & 1.402 & 1.416 & 1.420 & 1.426 & 1.435 & 1.435 & 1.452 & 1.459 & 1.460 & 1.479 \\
\hline$\mu\left(\mathrm{mm}^{-1}\right)$ & 0.369 & 0.373 & 0.374 & 0.375 & 0.378 & 0.378 & 0.382 & 0.384 & 0.384 & 0.389 \\
\hline Measd reflns & 3904 & 4265 & 4165 & 3487 & 10461 & 3256 & 8472 & 3408 & 3420 & 6135 \\
\hline Indep reflns & 2155 & 2145 & 2131 & 1584 & 2304 & 1580 & 1561 & 1554 & 1551 & 2136 \\
\hline $\mathrm{R}_{1}\left[\right.$ on $\left.^{2}{ }_{0}^{2}, \mathrm{I}>2 \sigma(\mathrm{I})\right]$ & 0.0700 & 0.0948 & 0.0925 & 0.0854 & 0.0877 & 0.1149 & 0.0875 & 0.1019 & 0.1034 & 0.1088 \\
\hline $\mathrm{wR}_{2}$ (all data) & 0.1218 & 0.2018 & 0.1923 & 0.1914 & 0.2345 & 0.2367 & 0.2163 & 0.2266 & 0.2125 & 0.3918 \\
\hline
\end{tabular}


Table 1b. Crystal data and details of measurements for crystalline $[1 \mathrm{H}]_{2} \mathrm{SO}_{4} \cdot \mathrm{H}_{2} \mathrm{O}(\mathrm{I}),[1 \mathrm{H}] \mathrm{BF} \mathrm{F}_{4}(\mathrm{I})$ and (II), $[\mathbf{1 H}] \mathrm{PF}_{6}$, and $[\mathbf{1 H}] \mathrm{NO}_{3}$.

\begin{tabular}{|c|c|c|c|c|c|}
\hline & {$[1 \mathrm{H}]_{2} \mathrm{SO}_{4} \cdot \mathrm{H}_{2} \mathrm{O}(\mathrm{I})$} & {$[1 \mathrm{H}] \mathrm{BF}_{4}(\mathrm{I})$} & {$[1 \mathrm{H}] \mathrm{BF}_{4}(\mathrm{II})$} & {$[1 \mathrm{H}] \mathrm{PF}_{6}$} & {$[1 \mathrm{H}] \mathrm{NO}_{3}{ }^{\mathrm{a}}$} \\
\hline Formula & $\mathrm{C}_{18} \mathrm{H}_{22} \mathrm{~N}_{2} \mathrm{O}_{9} \mathrm{~S}$ & $\mathrm{C}_{9} \mathrm{H}_{10} \mathrm{BF}_{4} \mathrm{NO}_{2}$ & $\mathrm{C}_{9} \mathrm{H}_{10} \mathrm{BF}_{4} \mathrm{NO}_{2}$ & $\mathrm{C}_{9} \mathrm{H}_{10} \mathrm{~F}_{3} \mathrm{NO}_{2} \mathrm{P}_{0.50}$ & $\mathrm{C}_{9} \mathrm{H}_{10} \mathrm{~N}_{2} \mathrm{O}_{5}$ \\
\hline fw & 442.44 & 250.99 & 250.99 & 236.67 & 226.06 \\
\hline Cryst. System & Monoclinic & Monoclinic & Triclinic & Triclinic & Monoclinic \\
\hline Space group & $\mathrm{P} 2{ }_{1} / \mathrm{c}$ & $\mathrm{I} 2 / \mathrm{a}$ & $\mathrm{P}-1$ & $\mathrm{P}-1$ & $\mathrm{P} 2{ }_{1} / \mathrm{c}$ \\
\hline $\mathrm{Z}$ & 4 & 8 & 2 & 2 & 4 \\
\hline $\mathrm{a}(\AA)$ & $13.1014(6)$ & $7.262(5)$ & $7.2802(11)$ & $5.5647(5)$ & $5.14519(1)$ \\
\hline $\mathrm{b}(\AA)$ & $11.7129(4)$ & $10.026(5)$ & $9.0670(15)$ & $5.8519(8)$ & $28.63202(6)$ \\
\hline $\mathrm{c}(\AA)$ & $13.2735(6)$ & $30.424(5)$ & 9.7329(16) & $15.8200(15)$ & $7.01640(2)$ \\
\hline$\alpha(\mathrm{deg})$ & 90 & 90 & $115.130(16)$ & $85.376(9)$ & 90 \\
\hline$\beta(\mathrm{deg})$ & $100.385(4)$ & $96.193(5)$ & $100.802(13)$ & $86.255(7)$ & $104.50484(5)$ \\
\hline$\gamma(\mathrm{deg})$ & 90 & 90 & $101.825(14)$ & $72.178(10)$ & 90 \\
\hline $\mathrm{V}(\AA 3)$ & $2003.52(15)$ & $2202.2(19)$ & $541.32(15)$ & $488.40(9)$ & $1000.699(1)$ \\
\hline Dcalc $(\mathrm{g} / \mathrm{cm} 3)$ & 1.467 & 1.514 & 1.540 & 1.609 & - \\
\hline$\mu(\mathrm{mm}-1)$ & 0.217 & 0.148 & 0.150 & 0.227 & - \\
\hline Measd reflns & 9219 & 5174 & 4239 & 3631 & - \\
\hline Indep reflns & 4638 & 2496 & 2441 & 2181 & - \\
\hline $\mathrm{R} 1[$ on $\mathrm{F} 02, \mathrm{I}>2 \sigma(\mathrm{I})]$ & 0.0761 & 0.0567 & 0.0857 & 0.0703 & - \\
\hline wR2 (all data) & 0.2476 & 0.1242 & 0.2686 & 0.1832 & - \\
\hline Rwp & - & - & - & - & 5.1 \\
\hline $\operatorname{Rexp}$ & - & - & - & - & 3.4 \\
\hline$\chi^{2}$ & - & - & - & - & 1.5 \\
\hline
\end{tabular}

\footnotetext{
${ }^{\mathrm{a}}$ Structural solution from powder data.
} 
Table 1c. Crystal data and details of measurements for crystalline $[1 \mathrm{H}]_{2} \mathrm{SO}_{4} \cdot \mathrm{H}_{2} \mathrm{O}$ (II) and for each stage of its photodimerization.

\begin{tabular}{|c|c|c|c|c|c|c|c|}
\hline & $\begin{array}{c}{[1 \mathrm{H}]_{2} \mathrm{SO}_{4} \cdot \mathrm{H}_{2}} \\
\mathrm{O} \\
(\mathrm{II}) \\
\end{array}$ & $\begin{array}{c}{[1 \mathrm{H}]_{2} \mathrm{SO}_{4} \cdot \mathrm{H}_{2}} \\
\mathrm{O} \\
\text { (II)_88\% }\end{array}$ & $\begin{array}{c}{[1 \mathrm{H}]_{2} \mathrm{SO}_{4} \cdot \mathrm{H}_{2}} \\
\mathrm{O} \text { (II)_70\% }\end{array}$ & $\begin{array}{c}{[1 \mathrm{H}]_{2} \mathrm{SO}_{4} \cdot \mathrm{H}_{2}} \\
\mathrm{O}(\mathrm{II})_{-} 58 \%\end{array}$ & $\begin{array}{c}{[1 \mathrm{HH}]_{2} \mathrm{SO}_{4} \cdot \mathrm{H}_{2}} \\
\mathrm{O}(\mathrm{III})_{-} 27 \%\end{array}$ & $\begin{array}{c}{[1 \mathrm{H}]_{2} \mathrm{SO}_{4} \cdot \mathrm{H}_{2}} \\
\mathrm{O} \text { (II)_20\% }\end{array}$ & $\begin{array}{c}{[1 \mathrm{HH}]_{2} \mathrm{SO}_{4} \cdot \mathrm{H}_{2}} \\
\mathrm{O}(\mathrm{II})_{-} 0 \%\end{array}$ \\
\hline Formula & $\begin{array}{c}\mathrm{C}_{18} \mathrm{H}_{22} \mathrm{~N}_{2} \mathrm{O}_{9} \\
\mathrm{~S}\end{array}$ & $\begin{array}{c}\mathrm{C}_{18} \mathrm{H}_{22} \mathrm{~N}_{2} \mathrm{O}_{9} \\
\mathrm{~S}\end{array}$ & $\begin{array}{c}\mathrm{C}_{18} \mathrm{H}_{22} \mathrm{~N}_{2} \\
\mathrm{O}_{9} \mathrm{~S}\end{array}$ & $\begin{array}{c}\mathrm{C}_{18} \mathrm{H}_{22} \mathrm{~N}_{2} \\
\mathrm{O}_{9} \mathrm{~S}\end{array}$ & $\begin{array}{c}\mathrm{C}_{18} \mathrm{H}_{22} \mathrm{~N}_{2} \\
\mathrm{O}_{9} \mathrm{~S}\end{array}$ & $\begin{array}{c}\mathrm{C}_{18} \mathrm{H}_{22} \mathrm{~N}_{2} \\
\mathrm{O}_{9} \mathrm{~S} \\
\end{array}$ & $\begin{array}{c}\mathrm{C}_{18} \mathrm{H}_{22} \mathrm{~N}_{2} \\
\mathrm{O}_{9} \mathrm{~S}\end{array}$ \\
\hline $\mathrm{fw}$ & 442.44 & 442.44 & 442.44 & 442.44 & 442.44 & 442.44 & 442.44 \\
\hline Cryst. System & Triclinic & Triclinic & Triclinic & Triclinic & Triclinic & Triclinic & Triclinic \\
\hline Space group & P-1 & P-1 & P-1 & P-1 & P-1 & P-1 & P-1 \\
\hline $\mathrm{Z}$ & 2 & 2 & 2 & 2 & 2 & 2 & 2 \\
\hline$a(\AA)$ & $7.3374(8)$ & $7.3066(9)$ & $7.2590(10)$ & $7.2497(10)$ & $7.1238(19)$ & $7.0960(5)$ & $7.0956(7)$ \\
\hline $\mathrm{b}(\AA)$ & $10.5566(12)$ & $10.5681(17)$ & $10.5719(15)$ & 10.6164(17) & $10.631(2)$ & $\begin{array}{l}10.6518(9) \\
\end{array}$ & $10.6597(13)$ \\
\hline$c(\AA)$ & $13.8798(15)$ & $13.8909(16)$ & $13.8895(18)$ & $13.902(2)$ & $13.925(3)$ & $13.9338(12)$ & $13.9430(15)$ \\
\hline$\alpha(\operatorname{deg})$ & 103.793(10) & $104.253(12)$ & $104.423(12)$ & $104.762(14)$ & $106.010(17)$ & $106.307(8)$ & $106.348(10)$ \\
\hline$\beta$ (deg) & 99.924(9) & $99.577(10)$ & $98.792(11)$ & $98.258(13)$ & $95.898(18)$ & $95.377(7)$ & 95.271(9) \\
\hline$\gamma(\mathrm{deg})$ & $102.838(10)$ & $102.609(12)$ & $102.601(12)$ & $102.574(13)$ & 102.081(19) & $101.948(7)$ & $101.923(10)$ \\
\hline$V\left(\AA^{3}\right)$ & 988.76(19) & $986.7(2)$ & 982.9(2) & 987.2(3) & $976.7(4)$ & 975.91(14) & 977.41(19) \\
\hline$D_{\text {calc }}\left(\mathrm{g} / \mathrm{cm}^{3}\right)$ & 1.486 & 1.489 & 1.495 & 1.488 & 1.504 & 1.506 & 1.503 \\
\hline$\mu\left(\mathrm{mm}^{-1}\right)$ & 0.219 & 0.220 & 0.221 & 0.220 & 0.222 & 0.222 & 0.222 \\
\hline Measd reflns & 8036 & 7514 & 7771 & 7361 & 7796 & 14762 & 7914 \\
\hline Indep reflns & 4494 & 4446 & 4480 & 4469 & 4453 & 4647 & 4462 \\
\hline $\begin{array}{c}\mathrm{R}_{1}\left[\mathrm{on} \mathrm{F}_{0}^{2}\right. \\
\mathrm{I}>2 \sigma(\mathrm{I})]\end{array}$ & 0.0681 & 0.0788 & 0.1020 & 0.1236 & 0.1032 & 0.1009 & 0.1076 \\
\hline $\mathrm{wR}_{2}$ (all data) & 0.1686 & 0.2483 & 0.3671 & 0.4059 & 0.3381 & 0.3155 & 0.2698 \\
\hline
\end{tabular}


Powder diffraction measurements. For phase identification purposes X-ray powder diffractograms in the $2 \theta$ range $5-40^{\circ}$ (step size, $0.02^{\circ}$; time/step, $20 \mathrm{~s} ; 0.04$ rad soller; $40 \mathrm{~mA} \mathrm{x} 40 \mathrm{kV}$ ) were collected on a Panalytical X'Pert PRO automated diffractometer equipped with an X'Celerator detector and in Bragg-Brentano geometry, using $\mathrm{Cu} \mathrm{K \alpha}$ radiation without a monochromator. The program Mercury ${ }^{2}$ was used for simulation of X-ray powder patterns on the basis of single crystal data. Chemical and structural identity between bulk materials and single crystals was always verified, when possible, by comparing experimental and simulated powder diffraction patterns, see Fig. ESI-1.
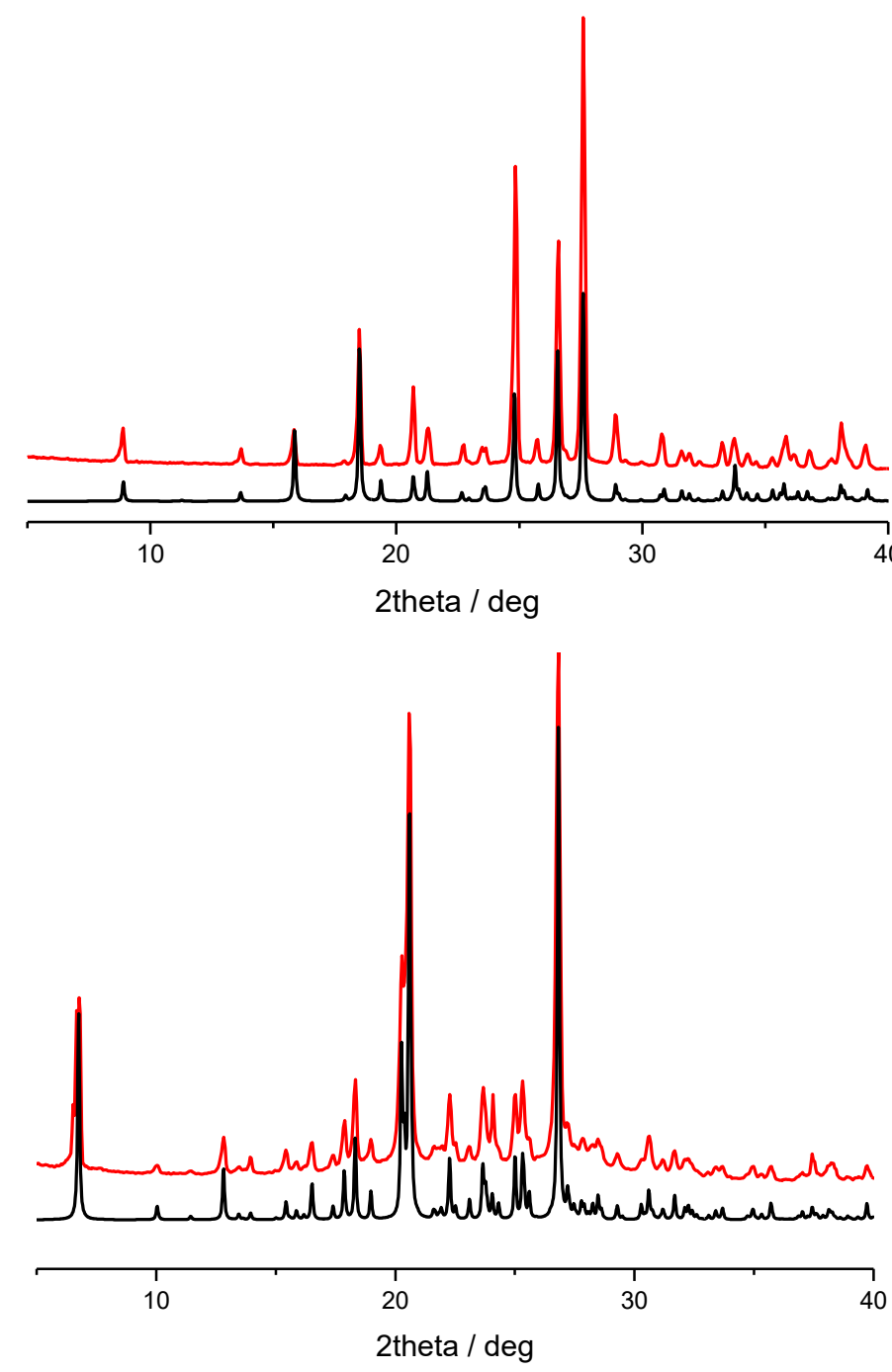

(a)

(b) 

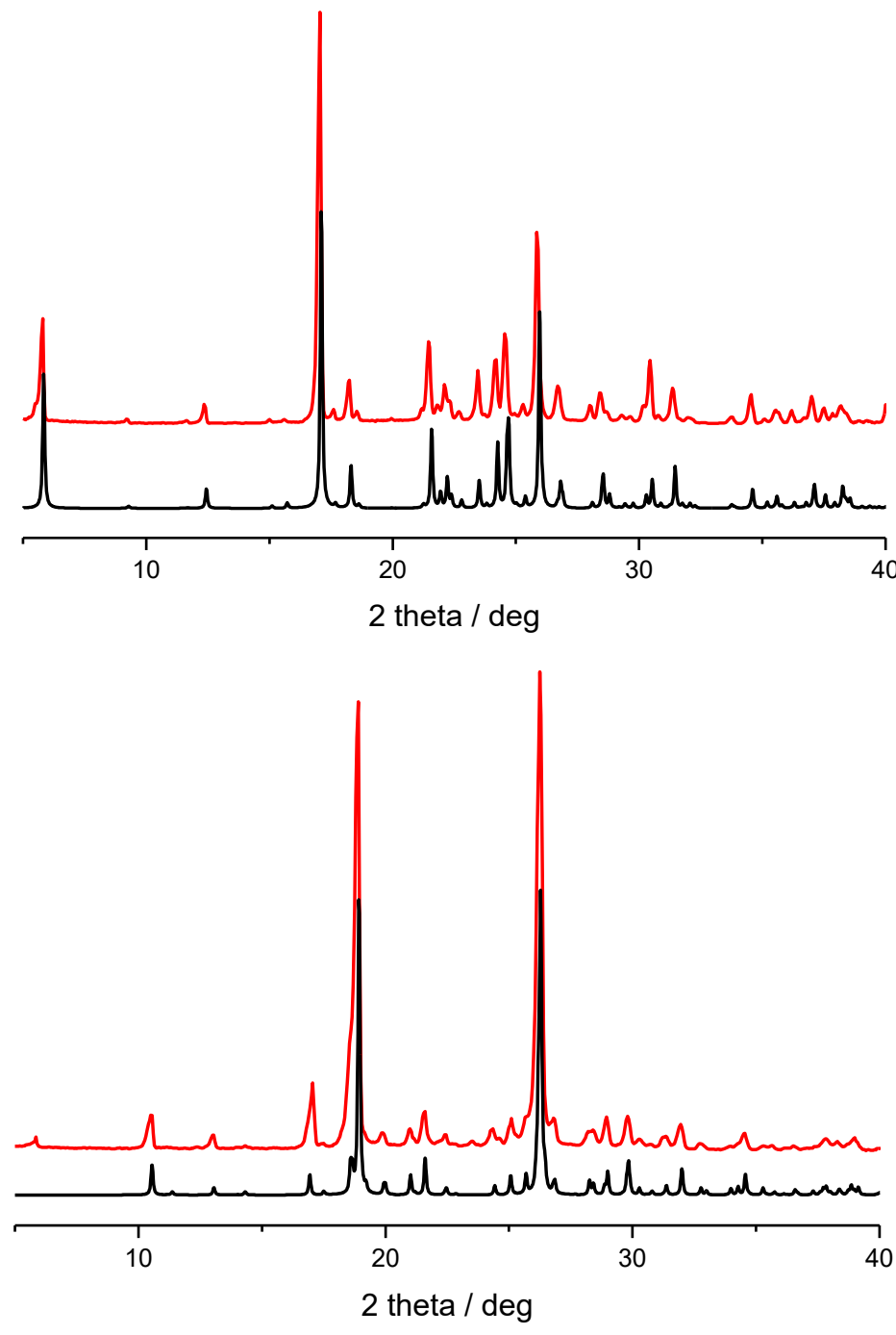

(c)

(d)



(e)

Fig. ESI-1. Experimental (red line) and simulated (black line) X-ray powder diffraction patterns for compounds: (a) $[\mathbf{1 H}] \mathrm{Cl},(\mathrm{b})[\mathbf{1}]_{2}\left[\mathrm{SO}_{4}\right] \cdot \mathrm{H}_{2} \mathrm{O}(\mathrm{I}),(\mathrm{c})[\mathbf{1 H}] \mathrm{BF}_{4}(\mathrm{I}),(\mathrm{d})[\mathbf{1 H}] \mathrm{BF}_{4}(\mathrm{II})$ and (e) $[\mathbf{1 H}] \mathrm{PF}_{6}$. 
Structure determination and refinement of $[1 \mathrm{H}] \mathrm{NO}_{3}$ from powder data. For structure solution and refinement purposes X-ray powder diffractograms in the $2 \theta$ range 5-60 (step size, $0.003^{\circ}$; time/step, 99s; $0.02 \mathrm{rad}$ soller; V x A $40 \times 40$ ) were collected on a Panalytical X'Pert PRO automated diffractometer operated in transmission mode (capillary spinner) and equipped with a Pixel detector.Powder diffraction data were analyzed with the software EXPO2010, ${ }^{8}$ which is designed toanalyze monochromatic andnon-monochromatic data. Peaks were automatically chosen inthe $2 \theta$ range $4-40^{\circ}$, and a monoclinic cell was found (seeTable $1 \mathrm{~b}$ ), using the algorithm NTREOR, ${ }^{9}$ with a volume of $1000.69 \AA^{3}$. The structure was then solved by simulated annealingusing amolecular model built with the Avogadro software, ${ }^{10}$ and refined by Rietveld method with thesoftware TOPAS4.1. ${ }^{11} \mathrm{~A}$ shifted Chebyshev function with 7 parameters and a Pseudo-Voigt function (TCHZ type) were used to fit backgroundand peak shape, respectively. A spherical harmonicsmodel was used to describe the preferred orientation. A rigid body was applied on the two fragments. Bond distances andtorsion angles were refined in a limited range around an equilibrium value.An overall thermal parameter for the $\mathrm{C}, \mathrm{N}, \mathrm{O}$ atoms was adopted. Refinement converged with $\chi^{2}=1.5, \mathrm{R}_{\mathrm{wp}}=5.1 \%, \mathrm{R}_{\mathrm{exp}}=3.4 \%$. Fig. ESI-2 shows experimental, calculated and difference curves.

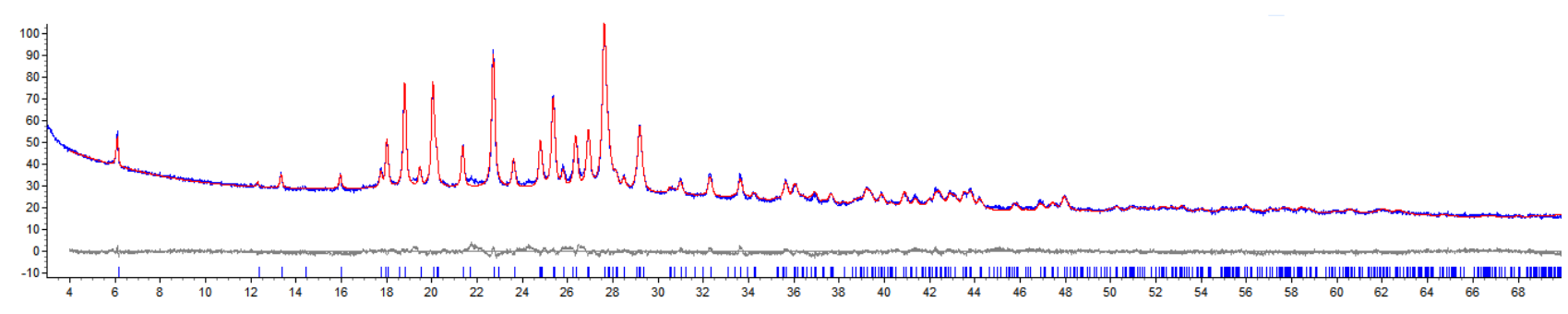

Fig. ESI-2. Experimental (blue), calculated (red) and difference (grey) patterns for $[\mathbf{1 H}] \mathrm{NO}_{3} ; \mathrm{x}$ axis is in degrees of $2 \theta$. 
Hydrogen bonds in $[1 \mathrm{H}] \mathrm{Cl}$. Compound $[1 \mathrm{H}] \mathrm{Cl}$ crystallizes in the monoclinic space group $\mathrm{P} 2{ }_{1} / \mathrm{c}$ with one $[\mathbf{1 H}]^{+}$and one $\mathrm{Cl}^{-}$ions in the asymmetric unit, and its crystal structure consists in a 2D network held together by hydrogen bonds between $[1 \mathrm{H}]^{+}$and $\mathrm{Cl}^{-}\left[\mathrm{NH}_{3}{ }^{+} \cdots \mathrm{Cl}^{-}=3.140(4)-3.194(4) \AA\right.$, $\mathrm{O}_{\mathrm{OH}} \cdots \mathrm{Cl}^{-}=3.056(3) \AA$, and $\mathrm{NH}_{3}{ }^{+} \cdots \mathrm{O}_{\mathrm{C}=\mathrm{O}}=2.801(4) \AA$ ], see Fig. ESI-3.

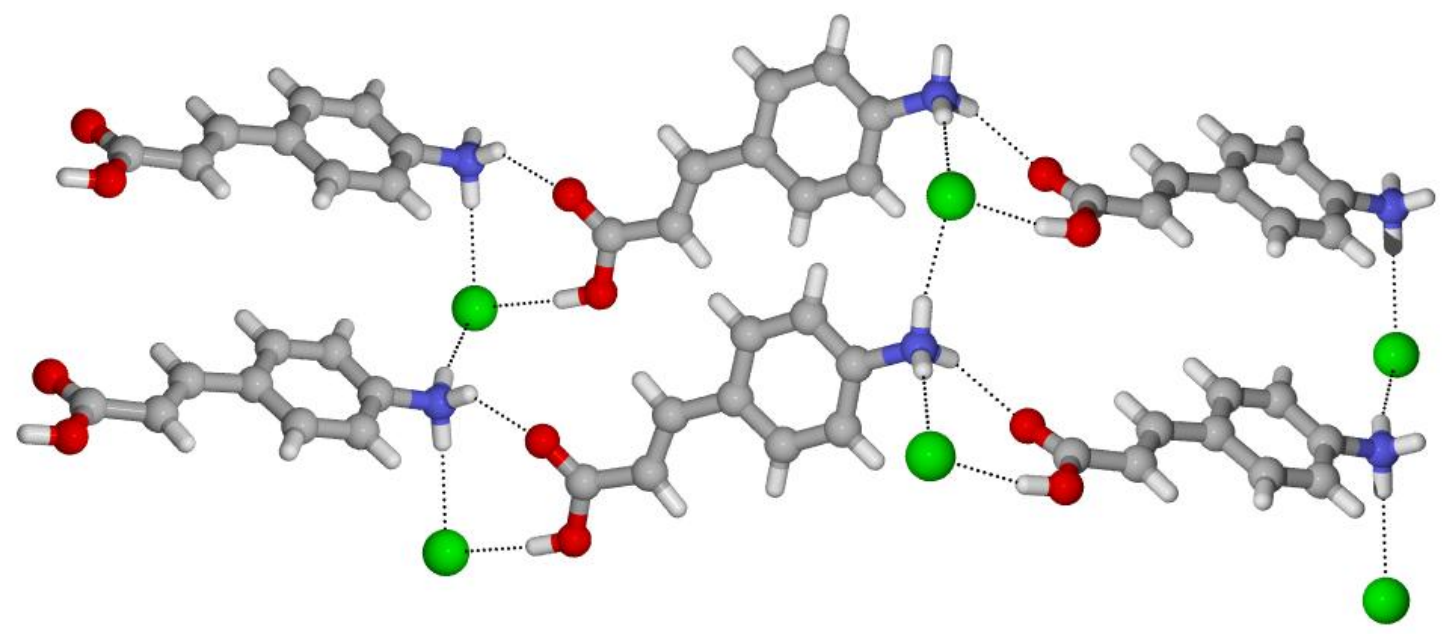

Fig. ESI-3.

Hydrogen bonds $\operatorname{in}[1 \mathrm{H}] \mathrm{NO}_{3}$. Compound $[1 \mathrm{H}] \mathrm{NO}_{3}$ crystallizes in the monoclinic space group $\mathrm{P}{ }_{1} / \mathrm{c}$ with one $[1 \mathrm{H}]^{+}$cation and one $\mathrm{NO}_{3}{ }^{-}$anionin the asymmetric unit, its structure features a 3D network of hydrogen bonds among the ionic species $[\mathbf{1 H}]^{+}$and $\mathrm{NO}_{3}{ }^{-}\left[\mathrm{NH}_{3}{ }^{+} \cdots \mathrm{NO}_{3}{ }^{-}=2.793(2)-2.932(3) \AA\right.$, $\mathrm{COOH} \cdots \mathrm{COOH}=2.677(2) \AA ̊]$, see Fig. ESI-4.

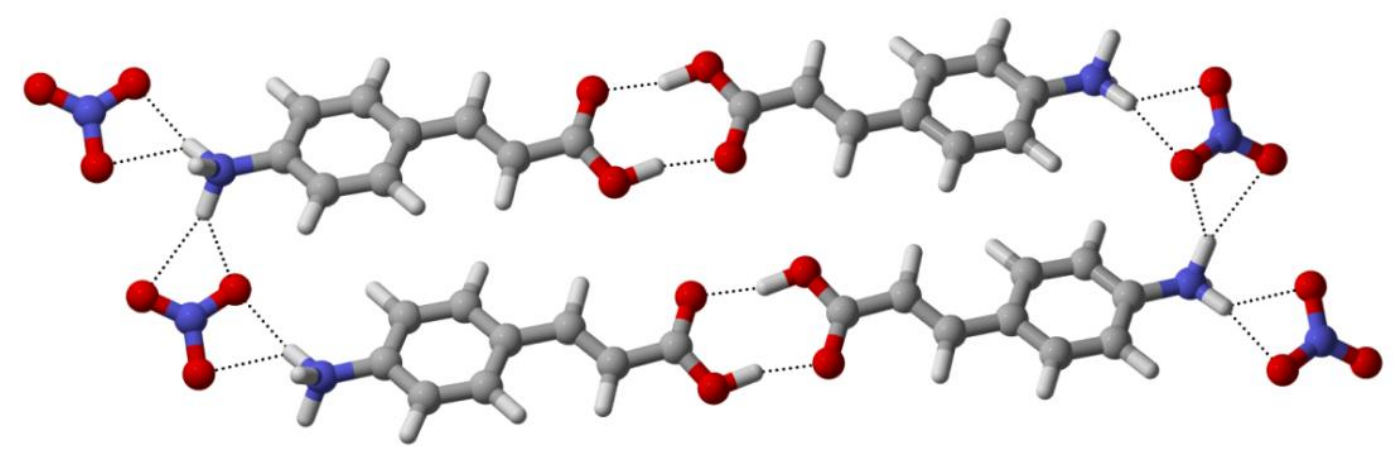

Fig. ESI-4. 
Hydrogen bonds in $\left[1 \mathrm{H}_{2}\left[\mathrm{SO}_{4}\right] \cdot \mathrm{H}_{2} \mathrm{O}(\mathrm{I}) \cdot[1 \mathrm{H}]_{2}\left[\mathrm{SO}_{4}\right] \cdot \mathrm{H}_{2} \mathrm{O}\right.$ (I) crystallizes in the monoclinic space group $\mathrm{P} 2{ }_{1} / \mathrm{c}$ with two $[1 \mathrm{H}]^{+}$one $\mathrm{SO}_{4}{ }^{2-}$ ions, and one water molecule in the asymmetric unit, its structure is characterized by the presence of a complex 3D network of hydrogen bonds between the ionic species $[1 \mathrm{H}]^{+}, \mathrm{SO}_{4}{ }^{2-}$ and the water molecule $\left[\mathrm{NH}_{3}{ }^{+} \cdots \mathrm{SO}_{4}{ }^{2-}=2.695(6)-2.805(6) \AA\right.$, $\mathrm{O}_{\mathrm{OH}} \cdots \mathrm{SO}_{4}{ }^{2-}=2.674(5)-2.778(7) \AA, \mathrm{O}_{\mathrm{w}} \cdots \mathrm{SO}_{4}{ }^{2-}=2.881(6) \AA, \mathrm{O}_{\mathrm{w}} \cdots \mathrm{O}_{\mathrm{C}=\mathrm{O}}=2.840(7) \AA, \mathrm{O}_{\mathrm{w}} \cdots \mathrm{NH}_{3}{ }^{+}=$ 2.724(7) $\left.\AA, \mathrm{NH}_{3}{ }^{+} \cdots \mathrm{O}_{\mathrm{C}=\mathrm{O}}=2.859(5) \AA ̊\right]$, see Fig. ESI-5.

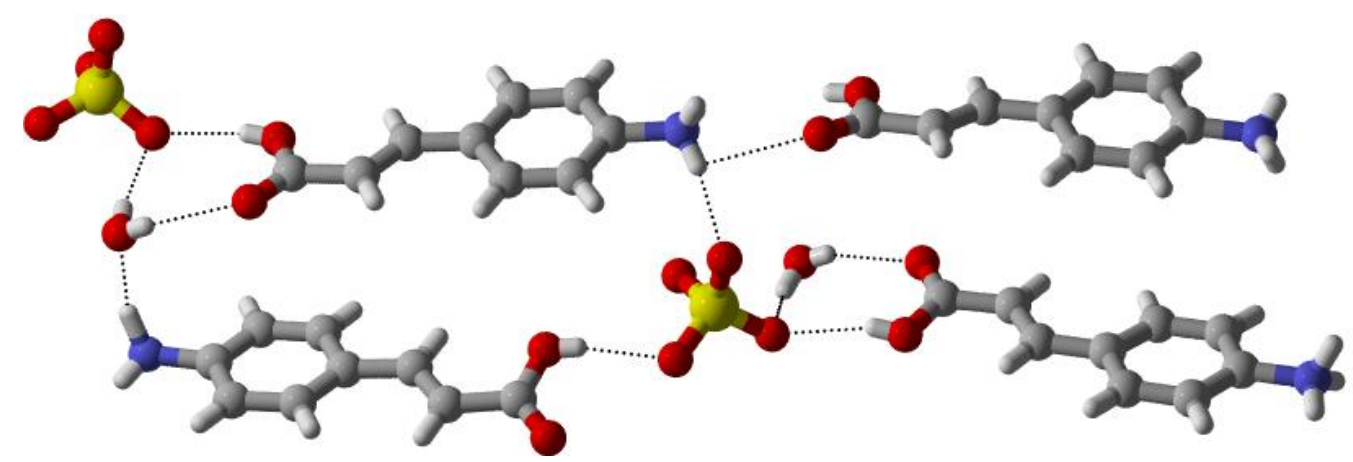

Fig. ESI-5.

Hydrogen bonds in $\left[1 \mathrm{H}_{2}\left[\mathrm{SO}_{4}\right] \cdot \mathrm{H}_{2} \mathrm{O}(\mathrm{II})\right.$. In crystalline $[1 \mathrm{H}]_{2} \mathrm{SO}_{4} \cdot \mathrm{H}_{2} \mathrm{O}$ (II), that crystallized in the triclinic space group $\mathrm{P}_{-1}$ with two $\mathbf{1 H}^{+}$cationic units and one $\mathrm{SO}_{4}{ }^{2-}$ anion and one water molecule in the asymmetric unit, there is a complex 3D network of hydrogen bonds among the components $\left[\mathrm{NH}_{3}{ }^{+} \cdots \mathrm{SO}_{4}{ }^{2-}=2.695(6)-3.053(5) \AA, \mathrm{O}_{\mathrm{OH}} \cdots \mathrm{SO}_{4}{ }^{2-}=2.612(3)-2.778(7) \AA, \mathrm{O}_{\mathrm{w}} \cdots \mathrm{SO}_{4}{ }^{2-}=2.781(7)-\right.$ 2.881(6) $\AA, \mathrm{O}_{\mathrm{w}} \cdots \mathrm{O}_{\mathrm{C}=\mathrm{O}}=2.840(7)-2.900(4) \AA, \mathrm{O}_{\mathrm{w}} \cdots \mathrm{NH}_{3}{ }^{+}=2.724(7)-2.797(5) \AA, \mathrm{NH}_{3}{ }^{+} \cdots \mathrm{O}_{\mathrm{C}=\mathrm{O}}=$ 2.850(4) - 2.859(5)Å] see Fig. ESI-6.

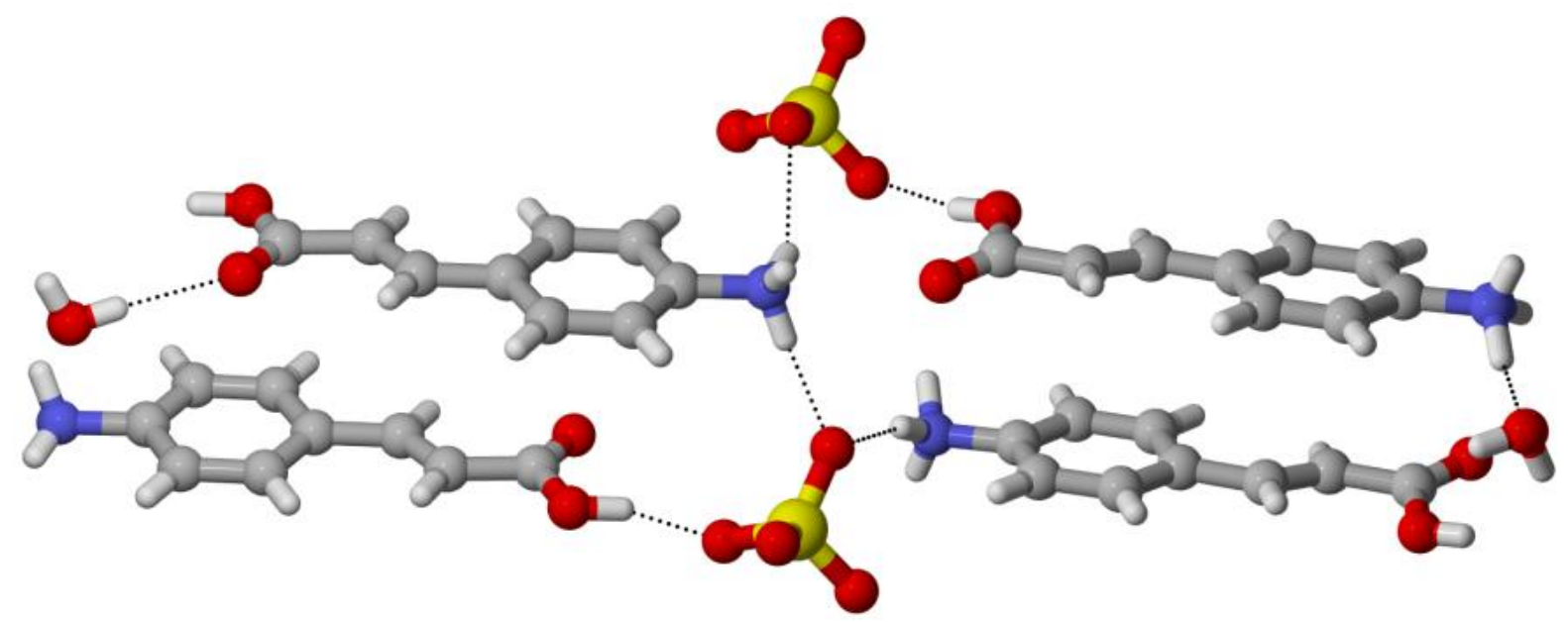

Fig. ESI-6. 
Hydrogen bonds in $[1 \mathrm{H}] \mathrm{BF}_{4}(\mathrm{I})$. Compound $[1 \mathrm{H}] \mathrm{BF}_{4}$ (I)crystallizes in the monoclinic space group I2/awith one $[1 \mathrm{H}]^{+}$cation and one $\mathrm{BF}_{4}{ }^{-}$anionin the asymmetric unit, its structure features a 3D network of hydrogen bonds between the ionic species $[1 \mathrm{H}]^{+}$and $\mathrm{BF}_{4}^{-}\left[\mathrm{NH}_{3}{ }^{+} \ldots \mathrm{BF}_{4}^{-}=2.732(4)\right.$ $3.038(4) \AA ̊, \mathrm{COOH} \cdots \mathrm{COOH}=2.625(4) \AA ̊]$, see Fig. ESI-7.

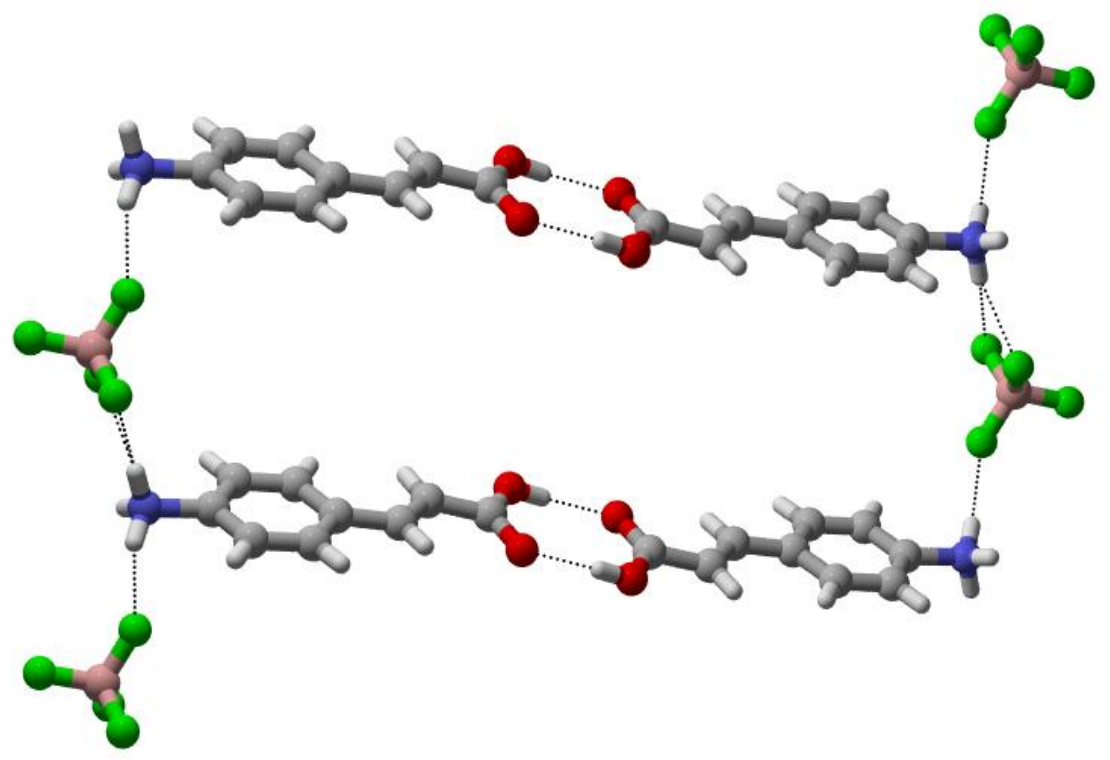

Fig. ESI-7.

Hydrogen bonds in $[1 \mathrm{H}] \mathrm{BF}_{4}(\mathrm{II})$. Compound $[1 \mathrm{H}] \mathrm{BF}_{4}$ (II)crystallizes in the triclinic space group $\mathrm{P}_{\text {. }}$ 1 with one $[1 \mathrm{H}]^{+}$cation and one $\mathrm{BF}_{4}{ }^{-}$anionin the asymmetric unit, its structure features a $3 \mathrm{D}$ network of hydrogen bonds between the ionic species $[1 \mathrm{H}]^{+}$and $\mathrm{BF}_{4}^{-}\left[\mathrm{NH}_{3}{ }^{+} \cdots \mathrm{BF}_{4}^{-}=2.987(6) \AA\right.$, $\left.\mathrm{C}=\mathrm{O} \cdots \mathrm{NH}_{3}{ }^{+}=2.829(5) \AA, \mathrm{O}-\mathrm{H}^{\prime} \cdots \mathrm{BF}_{4}{ }^{-}=2.789(7) \AA\right]$, see Fig. ESI-8.



Fig. ESI-8. 
Hydrogen bonds in $\left[1 \mathrm{H}_{\mathbf{P F}_{6}}(\mathrm{I})\right.$. Compound $[1 \mathrm{H}] \mathrm{PF}_{6}$ crystallizes in the triclinic space group $\mathrm{P}_{-1}$ with one $[\mathbf{1 H}]^{+}$cation and one $\mathrm{PF}_{6}{ }^{-}$anionin the asymmetric unit, its structure features a 3D network of hydrogen bonds between the ionic species $[1 \mathrm{H}]^{+}$and $\mathrm{BF}_{4}{ }^{-}\left[\mathrm{NH}_{3}{ }^{+} \ldots \mathrm{PF}_{6}{ }^{-}=2.717(4) \AA\right.$, $\mathrm{COOH} \cdots \mathrm{COOH}=2.634(5) \AA ̊]$, see Fig. ESI-10.

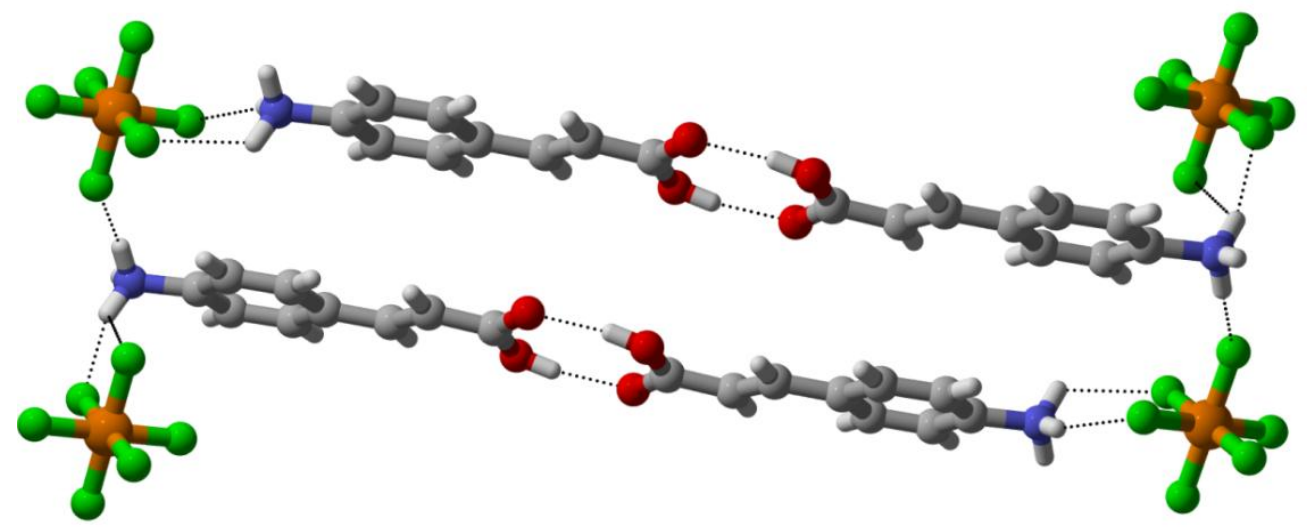

Fig. ESI-10.

Geometrical parameters used for the evaluation of photoreactivity. According to literature thereactivity for these salts is evaluated through the structural analysis of the geometrical parameters regarding the mutual orientation of neighboring $1 \mathrm{H}^{+}$cationic units, namely: $\mathrm{d}$ (distance between carbon atoms of $\mathrm{C}=\mathrm{C}$ bonds in adjacent moleculesknown as "Schmidt's rule", ${ }^{2,13}$ upper limit is $4: 2 \AA$ ), $\tau$ (torsion angle $\mathrm{C}=\mathrm{C} \cdots \mathrm{C}=\mathrm{C}$ formed by the adjacent molecules, ideal value is $0^{\circ}$ ), $\alpha$ (shift of one $\mathrm{C}=\mathrm{C}$ bond along the second $\mathrm{C}=\mathrm{C}$, ideal value is $90^{\circ}$ ), ${ }^{14}$ see scheme $\mathrm{ESI}-1$. Their values extracted from the crystal structures for all compounds are reported in Table ESI-2

Scheme ESI-1. Geometrical parameters used to evaluate the photoreactivity of all the crystalline compounds described in this study.
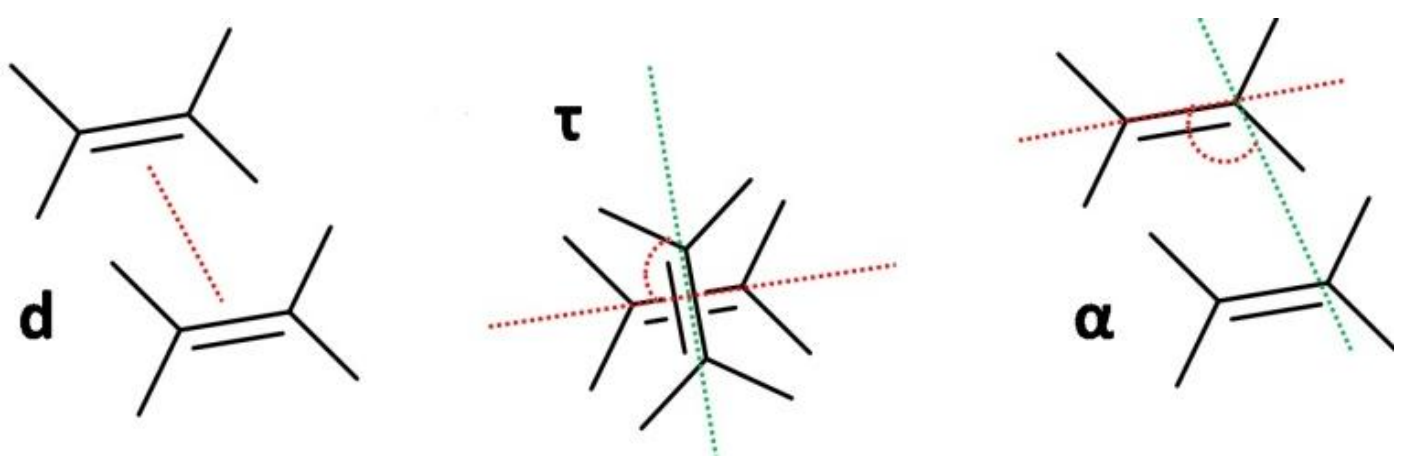
Table ESI-2. Types of arrangements observed in crystalline $[\mathbf{1 H}]_{\mathrm{n}} \mathrm{A} \cdot \mathrm{xH}_{2} \mathrm{O}$ where: $\mathbf{1}=4$-aminocinnamic acid, $\mathrm{A}^{\mathrm{n}-}=$ inorganic anion $\left(\mathrm{NO}_{3}{ }^{-}, \mathrm{BF}_{4}^{-}, \mathrm{PF}_{6}^{-}\right.$, and $\left.\mathrm{SO}_{4}{ }^{2-}\right) \mathrm{x}=1$, for the $\mathrm{SO}_{4}{ }^{2-}$, and $=0$, for all the other anions, together with their relevant geometrical parameters used for the evaluation of photoreactivity.

\begin{tabular}{lcccc} 
& type & $\mathbf{d}(\mathbf{A})$ & $\boldsymbol{\tau}\left({ }^{\circ}\right)$ & $\boldsymbol{\alpha}\left({ }^{\circ}\right)$ \\
\hline$[\mathbf{1 H}] \mathrm{Cl}$ & $\alpha^{\mathrm{a}}$ & $3.657(5)$ & 0 & $86.8(2)$ \\
{$[\mathbf{1 H}] \mathrm{NO}_{3}$} & $\alpha^{\mathrm{a}}$ & $4.128(2)$ & 0 & $86.8(5)$ \\
{$[\mathbf{1 H}]_{2}\left[\mathrm{SO}_{4}\right] \cdot \mathrm{H}_{2} \mathrm{O}(\mathrm{I})$} & $\alpha$ & $4.769(5)$ & $75.17(1)$ & $57.73(8)$ \\
& $\beta$ & $4.423(1)$ & $110.26(6)$ & $83.89(7)$ \\
{$[\mathbf{1 H}]_{2}\left[\mathrm{SO}_{4}\right] \cdot \mathrm{H}_{2} \mathrm{O}(\mathrm{II})$} & $\alpha 1^{\mathrm{a}}$ & $3.524(5)$ & 0 & $80.2(2)$ \\
& $\alpha 2$ & $4.726(6)$ & 0 & $56.7(2)$ \\
{$[\mathbf{1 H}] \mathrm{BF}_{4}(\mathrm{I})$} & - & - & & - \\
{$[\mathbf{1 H}] \mathrm{BF}_{4}(\mathrm{II})$} & $\alpha 1^{\mathrm{a}}$ & $3.681(7)$ & 0 & $74.4(3)$ \\
& $\alpha 2^{\mathrm{a}}$ & $3.746(3)$ & 0 & $88.6782)$ \\
{$[\mathbf{1 H}] \mathrm{PF}_{6}$} & $\beta$ & $5.565(1)$ & 0 & $56.72(3)$
\end{tabular}

Arrangements for two cationic units $1 \mathrm{H}^{+}: \alpha=$ head-to-tail; ${ }^{a}=$ double bonds related by an inversion center; $\beta$ = head-to-head; - = no arrangement.

Solid State Photoreactions. Single crystals samples were irradiatedusing a UV-LED (Led EnginLZ1-10UV00-0000) with $\lambda=365 \mathrm{~nm}$ and placed at a distance of $1 \mathrm{~cm}$. Powder samples were placedonto a flat spinner sample holder and irradiated using two Led EnginLZ1-10UV00-0000 $(\lambda=$ $365 \mathrm{~nm}$ ) placed at a distance of $2 \mathrm{~cm}$. X ray powder diffraction patterns were recorded every $4 \min$ and up to the completeness of the reaction. 
Hydrogen bond variations over the course of photoreaction of $[1 \mathrm{H}] \mathrm{Cl}$ and $\left[1 \mathrm{H}_{2} \mathrm{SO}_{4} \cdot \mathrm{H}_{2} \mathrm{O}(\mathrm{II})\right.$. During the course of the photoreaction is observed, in both cases, the loss of hydrogen bonds among the $\mathrm{COOH}$ and the $\mathrm{Cl}$ - and the water molecule for $[1 \mathrm{H}] \mathrm{Cl}$ and $[1 \mathrm{H}]_{2} \mathrm{SO}_{4} \cdot \mathrm{H}_{2} \mathrm{O}$ (II), respectively. For the latter this hydrogen bond is restored when reaction is completed, see Fig. ESI-11.

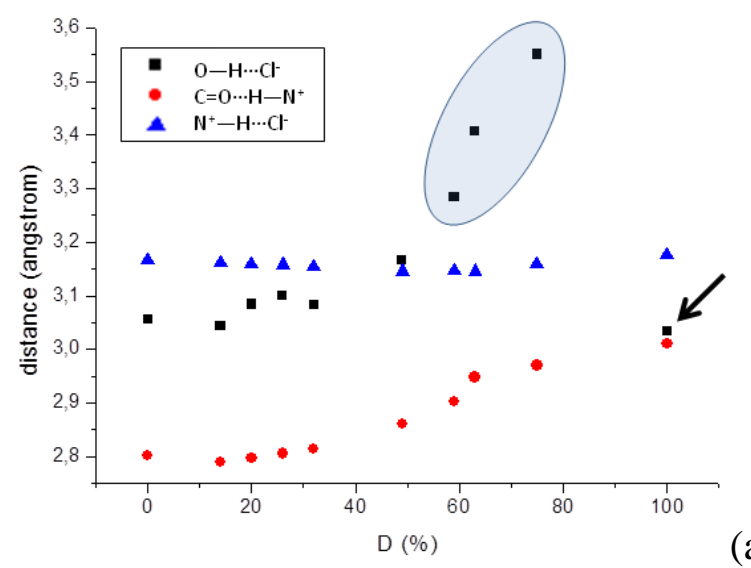

(a)

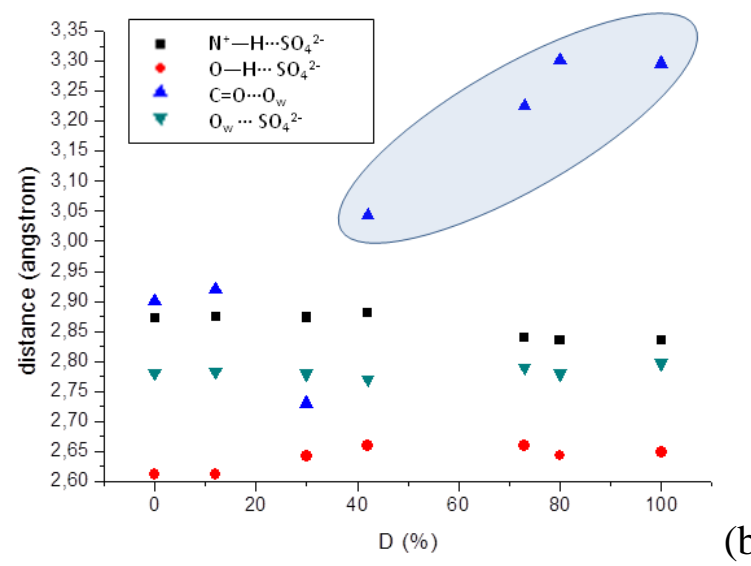

(b)

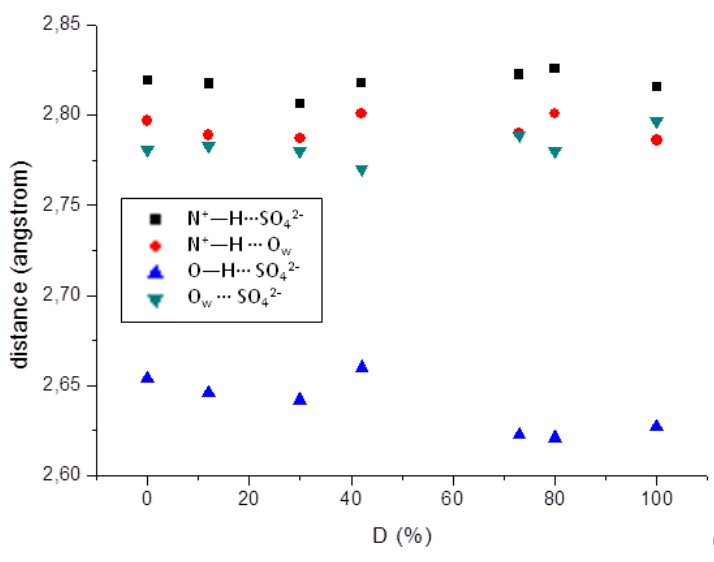

(c)

Fig. ESI-11.Variation of hydrogen bonding distances during the course of photoreaction in (a) $[1 \mathrm{H}] \mathrm{Cl}$, (b) $1 \mathrm{H}_{2} \cdot \mathrm{SO}_{4} \cdot \mathrm{H}_{2} \mathrm{O}$ (II) for the reactive arrangement $(\alpha 1)$, and (c) $\left[1 \mathrm{H}_{2} \mathrm{SO}_{4} \cdot \mathrm{H}_{2} \mathrm{O}\right.$ (II) for the no-reactive arrangement $(\alpha 2)$. The loss of hydrogen bonding interaction observed during the course of the reaction is highlighted with circles . 
Picture of single crystals of $[1 \mathrm{H}] \mathrm{Cl}$ and $\left[1 \mathrm{H}_{2}\left[\mathrm{SO}_{4}\right] \cdot \mathrm{H}_{2} \mathrm{O}\right.$ (II) before and after the photoreaction and crystal morphology for [1H]Cl.
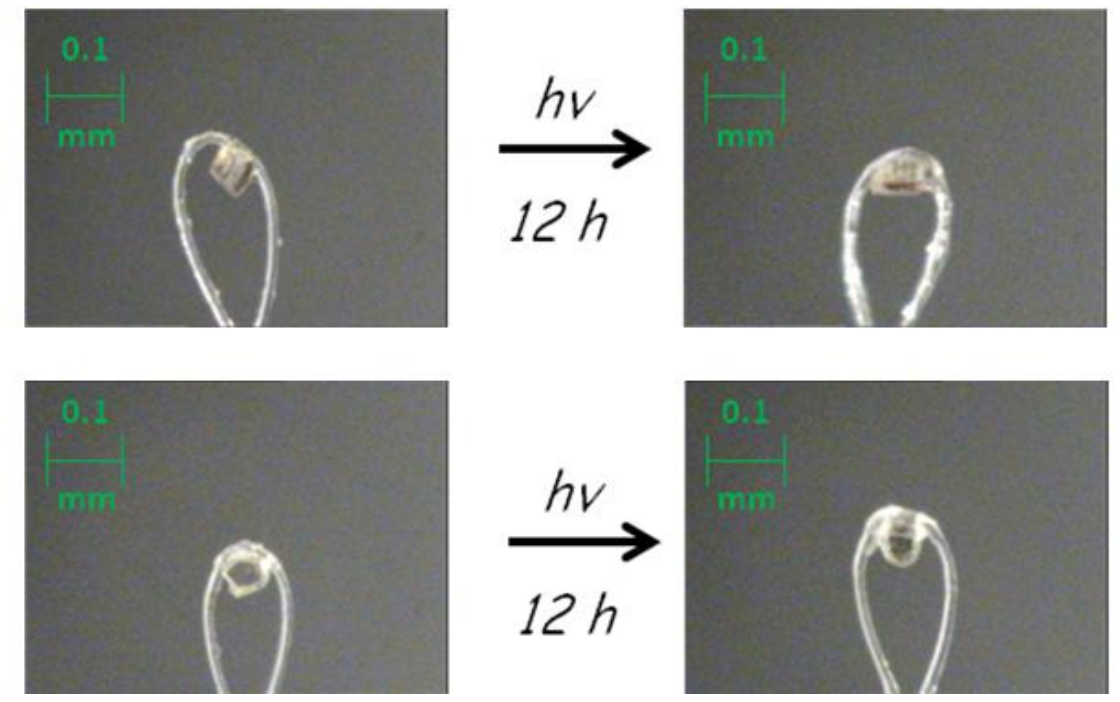

(b)

Fig. ESI-12. Pictures of single crystals before and after irradiation for: (a) $[\mathbf{1 H}] \mathrm{Cl}$ and (b) $[\mathbf{1}]_{2}\left[\mathrm{SO}_{4}\right] \cdot \mathrm{H}_{2} \mathrm{O}$ (II).

It should be also emphasized the relationship between crystal morphology and packing in $[1 \mathrm{H}] \mathrm{Cl}$, for which the face indexing was possible (due to the regular shape of the single crystals). Reactive double bonds are aligned almost perpendicularly to the crystallographic direction [010] and with the side groups lying in the $b c$-plane (Fig. ESI-13a). With the formation of the cyclobutane ring they bend outward; thus the major variation of the cell constants are expected along the $b$ - and $c$-axis ($3.67 \%$ and $-4.29 \%$, respectively) which are orthogonal to the direction of growth [100] (Fig. ESI13b) and also are the directions where the side groups movements provoke the greater strain and stress.

(a)


Fig.ESI-13. External morphology forsingle crystals of $[1 \mathrm{H}] \mathrm{Cl}$, (a) simulated BDFH on the basis of single crystal structure, and (b) experimentally determined after face indexing. In orange and blue are depicted the reactive $1 \mathrm{H}^{+}$ cationic units. 
Scanning Electron Microscopy. Morphological investigations of crystals were performed using a Philips XL-20 scanning electron microscope operating at $15 \mathrm{kV}$. The samples were observed asprepared and not sputter coated before examination. Sample preparation is as follows: single crystals were selected and, in order to remove the protective perfuoropolyether oil, washed up with ethyl acetate. For this reason, in some areas of SEM micrographs, it is possible to see on the crystal surface the presence of tiny residues of perfluoropolyether, especially for $[1 \mathrm{H}]_{2} \mathrm{SO}_{4} \cdot \mathrm{H}_{2} \mathrm{O}$ (II).

Plots of the variation of cell parameters during photoreaction for [1 $\mathrm{H}] \mathrm{Cl}$ and $[1 \mathrm{H}]_{2} \mathrm{SO}_{4} \cdot \mathrm{H}_{2} \mathrm{O}(\mathrm{II})$.

Fig. ESI-14 shows the variation of cell parameters, together with the cell volume, upon irradiation, for the same time intervals of Fig.4.


(a)

Fig. ESI-14. Variation of the unit cell constants and volume over the course of UV irradiation for $[\mathbf{1 H}] \mathrm{Cl}$ and $[1 \mathrm{H}]_{2}\left[\mathrm{SO}_{4}\right] \cdot \mathrm{H}_{2} \mathrm{O}(\mathrm{II})$.

The behaviour of these values clearly indicates that the photoreaction occurs via random cyclization of the cationic units in the crystals, not via crystal domains, and that the intermediate steps are representative of true solid solutions of monomers pairs and dimers. 
ATR-FTIR spectra of $[1 \mathrm{H}] \mathrm{Cl},[1 \mathrm{H}] \mathrm{NO}_{3}$ and $[1 \mathrm{H}] \mathrm{BF}_{4}$ (II) before and after $\mathrm{UV}$ irradiation. Attenuated total reflectance Fourier transform IR (ATR-FTIR) spectra were obtained using a Bruker Alpha FT-IR spectrometer.ATR-FTIR spectra were run on polycrystalline samples of $[1 \mathrm{H}] \mathrm{Cl},[1 \mathrm{H}] \mathrm{NO}_{3}$, and $\left[1 \mathrm{H}^{-\mathrm{BF}_{4}}\right.$ (II) before and after overnight irradiation at $365 \mathrm{~nm}$ (Fig. ESI-15). Only in the case of compound $[1 \mathrm{H}] \mathrm{Cl}$, the characteristic band of the conjugated $\mathrm{C}=\mathrm{O}$ stretching at $1690 \mathrm{~cm}^{-1}$ shifted to $1710 \mathrm{~cm}^{-1}$ due to the deconjugation of the carbonyl group and the disappearance of olefinic $\mathrm{C}=\mathrm{C}$ stretching $\left(1639 \mathrm{~cm}^{-1}\right)$ after the $[2+2]$ photodimerization. These changes are in agreement with what observed by Chu and co-workers for similar systems. ${ }^{15,16}$

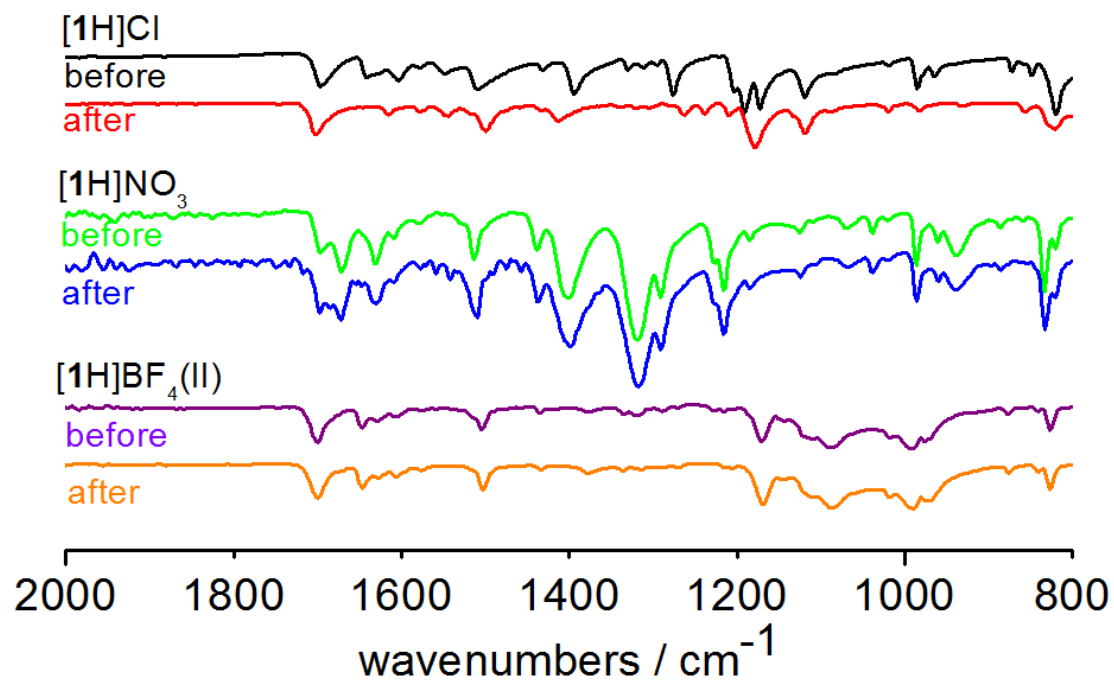

Fig. ESI-15. ATR-FTIR spectra of compounds $[1 \mathrm{H}] \mathrm{Cl},[\mathbf{1 H}] \mathrm{NO}_{3}$, and $[\mathbf{1 H}] \mathrm{BF}_{4}$ (II) recorded before and after irradiation $(365 \mathrm{~nm})$ overnight.

NMR spectroscopy. ${ }^{1} \mathrm{H}-\mathrm{NMR}$ spectra were recorded on a Varian INOVA $400(400 \mathrm{MHz})$ spectrometer and using, as solvent, dimethylsulfoxyde- $\mathrm{d}_{6}\left(\mathrm{DMSO}-\mathrm{d}_{6}\right)$ bought from Sigma-Aldrich. Chemical shifts are reported in ppm using tetramethylsilane as the internal reference standard. Data are reported as follows: chemical shift, multiplicity $(\mathrm{d}=$ doublet, $\mathrm{dd}=$ doubledoublet $)$, coupling constants $(\mathrm{Hz})$, and number of protons $(\mathrm{nH})$. Spectra were run on polycrystalline samples of $[1 \mathrm{H}] \mathrm{Cl}$ before and after overnight irradiation at $365 \mathrm{~nm}$. Completion of the [2+2] photoreaction is confirmed by the disappearing of the olefin peaks at 7.5 and $6.4 \mathrm{ppm}$ and the emergence of cyclobutane protons in at 4.3 and $3.8 \mathrm{ppm}$.

Before irradiation, $[1 \mathrm{H}] \mathrm{Cl}\left(\mathrm{DMSO}_{-} \mathrm{d}_{6}, 400 \mathrm{~Hz}\right): 7.66(\mathrm{~d}, J=8.2 \mathrm{~Hz}, 2 \mathrm{H}), 7.54(\mathrm{~d}, \mathrm{~J}=16 \mathrm{~Hz}, 2 \mathrm{H})$, $7.15(\mathrm{~d}, J=8.2 \mathrm{~Hz}, 1 \mathrm{H}), 6.43(\mathrm{~d}, \mathrm{~J}=16 \mathrm{~Hz}, 1 \mathrm{H})$.

After irradiation[ $\left.\mathbf{1}_{2} \mathrm{H}_{2}\right] \mathrm{Cl}_{2}\left(\mathrm{DMSO}_{\mathrm{d}}, 400 \mathrm{~Hz}\right): 7.47(\mathrm{~d}, J=8.2 \mathrm{~Hz}, 4 \mathrm{H}), 7.34(\mathrm{~d}, \mathrm{~J}=8.2,4 \mathrm{H}), 4.31$ $(\mathrm{dd}, J=10 \mathrm{~Hz} / 7.5 \mathrm{~Hz}, 2 \mathrm{H}), 3.84(\mathrm{dd}, \mathrm{J}=10 \mathrm{~Hz} / 7.5 \mathrm{~Hz}, 2 \mathrm{H})$. 


\section{References}

[1] Sheldrick, G. M. SHELX97, Program for Crystal Structure Determination; University of Göttingen: Göttingen, Germany, 1997.

[2] C. F. Macrae, I. J. Bruno, J. A. Chisholm, P. R. Edgington, P. McCabe, E. Pidcock, L. Rodriguez-Monge, R. Taylor, J. van de Streek and P. A. Wood, J. Appl. Crystallogr., 2008, 41, 466.

[3] C. Y. Legault, CYLview, Université de Sherbrooke, 2009.

[4] V. Enkelmann and G. Wegner, J. Am. Chem. Soc., 1993, 115, 10390.

[5] F.-L. Hu, S.-L. Wang, J.-P. Lang and B. F. Abrahams, Sci. Rep., 2014, 4, Article number: 6815.

[6] I. Turowska-Tyrk, Acta Cryst., 2003, B59, 670).

[7] G. Kumar Kole, T. Kojima, M.Kawano, and J. J. Vittal, Angew. Chem. Int. Ed., 2014, 53, 1.

[8] A. Altomare, M. Camalli, C. Cuocci and R. Rizzi, J. Appl. Crystallogr., 2009, 42, 1197.

[9] A. Altomare, C. Giacovazzo, A. Molinterni and R. Rizzi, J. Appl. Crystallogr., 2001, 34, 704.

[10] Avogadro: an open-source molecular builder and visualization tool. M. D. Hanwell, D. E. Curtis, D. C. Lonie, T. Vandermeersch, E. Zurek and G. R. Hutchison, J. Cheminf., 2012, 47.

[11] A. Cohelo, TOPAS-Academic, Coelho Software, Brisbane, Australia, 2007.

[12] M. D. Cohen, G. M.Schmidt, J. Am. Chem. Soc., 1964, 2000.

[13] G. M. Schmidt, J. Pure Appl. Chem. 1971, 27, 647-678.

[14] S.-Y. Yang, P. Naumov, and S.Fukuzumi, J. Am. Che. Soc., 2009, 131, 7247.

[15] Z. Wang, K. Randazzo, X. Hou, J. Simpson, J. Struppe, A. Ugrinov, B. Kastern, E. Wysocki, and Q. R. Chu, Macromolecules, 2015, 48, 2894.

[16] Z. Wang, B. Kastern, K. Randazzo, A. Ugrinov,J. Butz, D. W. Seals, M. P. Sibi and Q. R. Chu, GreenChem., 2015, 17, 4720.

[17] W. Yang, Yanchang Gan, X. Jiang and Hongzhi Li, Eur. J. Inorg. Chem., 2015, 99. 